\title{
28 Research Soure \\ Efficacy and Outcomes of a Novel Telephone-based Gout Disease Management Program
}

\author{
Abdalhamid Al Harash \\ Allegheny Health Network https://orcid.org/0000-0001-9849-7235 \\ Tarun Sharma \\ Allegheny Health Network \\ Gina Laginya \\ Allegheny Health Network \\ Mary Chester M Wasko ( $\nabla$ marychester.wasko@ahn.org ) \\ Allegheny Health Network \\ William T Ayoub \\ Allegheny Health Network
}

Research article

Keywords: Gout, rheumatology, telephone-based management

Posted Date: June 24th, 2020

DOI: https://doi.org/10.21203/rs.3.rs-36000/v1

License: (c) (1) This work is licensed under a Creative Commons Attribution 4.0 International License.

Read Full License 


\section{Abstract}

Objective: Gout is the most common inflammatory arthritis in the United States. Despite published guidelines, management remains suboptimal, leading to unnecessary morbidity and increased cost of care. We have designed the gout disease management program (GDMP) to improve outcomes, increase patient satisfaction and decrease healthcare utilization.

Methods: Gout patients were seen at their usual rheumatology clinical visit and offered participation in the GDMP. Data were collected between April 2017 and May 2019. Serum uric acid (SUA) levels were measured at initial outpatient encounter, at entrance to GDMP and every 4 weeks until SUA was at goal of $\leq 6 \mathrm{mg} / \mathrm{dl}$. During telephone encounters, gout-related recent hospitalizations and ER or urgent care visits since last encounter were ascertained. Self-reported gout medication usage and adherence were also determined. Patient satisfaction with GDMP was surveyed using a 5-point Likert scale.

Results: A total of 158 patients were enrolled, of which $112 \mathrm{had} \geq 1$ telephone encounter and were included in our analyses. During the telephone phase, 79 patients $(70 \%)$ achieved SUA goal of $\leq 6.0$ $\mathrm{mg} / \mathrm{dl}$, while90 patients $(80 \%)$ achieved an acceptable SUA of $\leq 6.5 \mathrm{mg} / \mathrm{dl}$. Only 3 patients $(2.6 \%)$ required hospitalization or visits to an ER or urgent care center due to gout flare, and $98 \%$ rated their encounter as a 5 on the 5 -point Likert scale.

Conclusion: Our telephone-based management program for gout led to improved clinical outcomes as defined by the ACR guidelines, decreased healthcare visits, and had high patient satisfaction.

\section{Significance And Innovations}

- First telephone-based, rheumatology providers-led study to manage gout · First study to show excellent patient satisfaction

\section{Introduction}

While gout is an ancient and common inflammatory arthritis, its management remains a clinical challenge (1). In the United States, controversies regarding treatment strategies are evident in the guidelines of two major institutions. The recently updated American College of Rheumatology guidelines consistently advise a treat-to-target approach, with a goal serum uric acid (SUA) of $£ 6 \mathrm{mg} / \mathrm{dl}(2,3)$. The American College of Physiciansrecommendsbasing treatment intensity on minimizing symptoms rather than a SUA goal (4).

Regardless of theguideline applied,evidence indicates that long-term management of gout is infrequently optimized. Treatmentsthat lower SUA arenot used regularly, are inconsistently prescribed, and are often under-dosed (5). Furthermore, patient compliance remains a challenge. First, gout is an episodic disease with long asymptomatic periods between attacks, giving patients the sense that they are well enough to not take their medication. Additionally,limited access to rheumatology services or time needed away from 
work in order to travel to a clinic can deter patients from remaining adherent to therapy. Furthermore, lack of patient education regarding gout has been shown to affectcompliance (6).

Pharmacist- and nurse-led telemedicine programs aimed at improving gout-related outcomes havebeen shown to not only improve medication compliance but have also demonstrated effectiveness in increasing the proportion of patients achieving goal SUA level of $£ 6 \mathrm{mg} / \mathrm{dl}(7,8)$. However, to date, healthcare utilization and patient satisfaction with telemedicine programs havenot been studied. We designed and instituted a gout disease management program (GDMP) in which the management of gout is initially performed ata standard office visit,and then followed up with a novel telephone-based management program, in which further management with patient education can take place.The objective of the GDMP was to increase the proportion of patients reaching targetSUA goal with resultant clinical control of gout attacks, thus minimizing the need for hospitalization or emergency/urgent care center visits while achieving patient satisfaction.

\section{Materials \& Methods}

The GDMPwas designed to provide longitudinal care for patients after their usual clinical visits with a rheumatology provider. The treating rheumatologist made the decision to enroll the patient into GDMP at the initial visit or follow-up visits if the patient was agreeable. The first patient was enrolled in April 2017, and patients were followed until November 2019. Patients were enrolled regardless of their baseline SUA, and there were no exclusion criteria. This was determined to be an IRB-exempt project, therefore written patient consent was not obtained.

\section{Gout Definition, Telephone Encounters, and Data Collection}

Gout was initially diagnosed by the referring rheumatology provider who also managed and diagnosed flares. A rheumatologistor mid-level rheumatology provider then followed up with the patient, performing periodic SUA levels and telephone encounters. The telephone encounters were conducted monthly until SUA level was at goal of $£ 6 \mathrm{mg} / \mathrm{dl}$, and patients were flare free for 3 months (or 6 months for tophaceous gout), then every 6 monthsfor 2 encounters, and finally, yearly if stable.

During the telephone encounters,recent flare and hospitalizations or emergency care visits due to gout since last encounter were recorded. Additionally, patient's gout-related medication usage and adherence were monitored by self-report during each phone call. Side effects of medications were documented and patient satisfaction with the telephone encounters versus usual care office visits was surveyed using a 5point Likert scale. If a telephone encounter could not be completedeasily, a secure email messagewith the same questions was sent to the patient usingelectronic health records.Ifboth methods wereunsuccessful, an instructional letter was mailedto the patient to contact theirrheumatology provider. Patients did not incur any charge for the telephone encounters.

\section{Urate Lowering Therapy and Prophylactic Medications}


Allopurinol was used as the first-lineurate lowering therapy (ULT) of choice. In patients that could not tolerate allopurinol, febuxostat was used instead. Allopurinol starting dose was $100 \mathrm{mg}$ daily in patients with an estimated glomerular filtration rate (eGFR) ${ }^{3} 60 \mathrm{ml} / \mathrm{min}$ and $50 \mathrm{mg}$ daily in patients witheGFR£60 $\mathrm{ml} / \mathrm{min}$. If patients were not already taking ULT, it was initiated 2 weeks after an acute flare resolved. Allopurinol doses were increased by $100-\mathrm{mg}$ increments in patients witheGFR $60 \mathrm{ml} / \mathrm{min}$ and $50-\mathrm{mg}$ increments in patientswith eGFR $60 \mathrm{~mL} / \mathrm{min}$ until a goal of SUA $£ 6 \mathrm{mg} / \mathrm{dl}$ was achieved. In patients receiving febuxostat, starting dose was $40 \mathrm{mg}$ daily, which could be increased to a maximum of $80 \mathrm{mg}$ daily.Probenecid, at a dose of $500 \mathrm{mg}$ twice daily,was used if both allopurinol and febuxostat were contraindicated.

Colchicine, nonsteroidal anti-inflammatory drugs, or glucocorticoids were used as prophylactic medications to suppress gout attacks with institution and dose adjustment of ULT. Oncetarget SUAwas achieved and maintained for 3 months (or 6 months for tophaceous gout), prophylactic medications were discontinued. Patients were allowed to be on ULT and/or prophylactic medications prior to entering into the GDMP, but doses were adjusted based on the above as needed.

\section{Laboratory Monitoring}

Complete blood count, creatinine and liver function tests were obtained at baseline and after 3 months. SUA levels were measured in all patients at their initial outpatient office rheumatology visit, at the time of entry into the telephone phase, and every 4 weeks until SUAwas $\leq 6 \mathrm{mg} / \mathrm{dl}$.

\section{Patient Education and Medications Refills}

Patient education (treat-to-target approach) was provided at the first visit with their office providers and with subsequent telephone calls. Medication refills were submitted electronically to the patient's pharmacy, and SUA laboratory requisitions were sent to the patient by mail or electronically to the lab.

\section{Results}

As of November 2019, 158 patients were enrolled inthe GDMP. A total of 112 patients who had $\geq 1$ followup in the telephone phaseare included here (some patients are not reported here due to the lack of a telephone encounter at the time of data extraction). Mean age at GDMP enrollment was 60years, and $87 \%$ were males. Twenty-six patients $(23 \%)$ had a crystal-proven diagnosis, while tophaceous gout was diagnosed in 31 patients (27\%). A phone call took approximately 5-10 minutes to complete. At least one flare was reported in 25 patients (22\%) after entry into the program.

After the initial visit with a rheumatology provider, 108 patients initiated ULT along with appropriate prophylaxis (4 patients either refused ULT or discontinued it without medical input). Ninety-one patients $(81 \%)$ were treated with allopurinol,16 (14\%) withfebuxostat, and 1 with probenecid. Colchicine was the most commonly used medication for prophylaxis $(n=41,37 \%)$. 
Prior to their firstrheumatology visit, 25 patients(22\%) had a SUA $\leq 6.0 \mathrm{mg} / \mathrm{dl}$ (Figure 1). At entry in the telephone phase, 47 patients $(41 \%)$ had SUA of $\leq 6.0 \mathrm{mg} / \mathrm{dl}$, and by the end of the GDMP observation phase, 79 patients $(71 \%)$ had achieved SUA goal of $\leq 6.0 \mathrm{mg} / \mathrm{dl}$. Of note, 90 patients $(80 \%)$ achieved an acceptable SUA level of $\leq 6.5 \mathrm{mg} / \mathrm{dl}$ with good clinical control of their disease by the end of the observation phase. Figure 2 further delineates and compares the percentage of patients achieving SUA levels, by $0.5 \mathrm{mg} / \mathrm{dl}$ intervals, at the start of the GDMP vs at the end of the study. This figure demonstrates a shift in the distribution to the left, reflecting an increased number of patients with SUA $\leq 6.0 \mathrm{mg} / \mathrm{dl}$.

Prior to entry into GDMP, 25 patients (22\%) had required emergency or urgent care services due to gout, and 10 patients (9\%) had beenhospitalized due to gout (Figure 3). During participation in the GDMP, only 3 patients $(2.6 \%)$ required an emergency department or urgent care center visit due to gout flare, and none required hospitalization.

Patients were extremely satisfied with the telephone encounters; $98 \%$ of patients rated their encounter as a 5 on a 5-point Likert scale.

\section{Discussion}

We report an effective and well-received follow-up program delivered by telemedicine for medication adjustment and monitoring in the longitudinal management of gout. The goal SUA $\leq 6 \mathrm{mg} / \mathrm{dl}$ was achieved in $>70 \%$ of patients, and $80 \%$ of the patient's achieved a more lenient SUA of $\leq 6.5 \mathrm{mg} / \mathrm{dl}$ with good clinical control of their symptoms. Additionally, the majority of patients were on appropriate prophylactic medications while adjusting the dose of ULT, thereby reducing the likelihood of gout flares. There were no hospitalizations and very few ER or urgent care visits during the observation period of the program. Some patients who did not achieve the goal were likely due to inadequate time in the telephone phase of the study before the study came to a close, inability to reach the patient via phone or other communication methods, and patient noncompliance with medication.

Our program was designed to overcome many obstacles in the longitudinal treatment of gout. Patients were monitored by a series of telephone calls, thus offering convenience to the patient. The provider reinforced medication adherence during the telephone calls and repeat SUA levels were performed regularly. Patient education was provided, which might have led to better compliance and more frequent achievement of SUA target. Medication refills were sent electronically with telephone encounters, and we used electronic medical record to contact the patient through messages in cases when phone calls were unavailable, an additional convenience to the patients.

Access to care remains a major issue in rheumatic diseases in the United States due to a nationwide shortage of practitioners. Our GDMP is an ideal way to limit bedside outpatient visit utilization and optimize regular follow-up with a focus on optimizing SUA, targeting a level of $\leq 6 \mathrm{mg} / \mathrm{dl}$ to reduce the likelihood of gouty attacks and long-term consequences of hyperuricemia. Gout is an ideal disease that 
lends itself to treatment algorithms and telephone-based care with application of available management recommendations.

The guidelines from the American College of Rheumatology and the American College of Physicians are available for gout treatment $(2,3,4)$.Nevertheless, gout treatment remains suboptimal. The reason for this shortfallis likely multifactorial. The lack of symptoms between gout flares decreases patient adherence to chronic daily ULT medications. Additionally, the practitioner's concern of medication toxicity can lead to using less optimum doses of medication.Furthermore, the frequent need to adjust the dose of medications and obtain SUA level (which typically requires multiple office visits) is an obstacle to many patients due to time constraints. Lastly, the treat-to-target approach, which is adopted by most rheumatologists as advocated by the ACR guidelines $(2,3)$, is not necessarily embraced by primary care providers based on the prior 2017 ACP guidelines (4).

Similarly to our study, inthe RamP-UP study, patients were contacted directly by pharmacists(7). The SUA goal of $\leq 6 \mathrm{mg} / \mathrm{dl}$ was achieved in $30 \%$ of patients in the intervention group, in comparison to only $15 \%$ in the usual care group. Adherence was also higher in the intervention group. Intervention period was one year only. Our results differedfrom the RAmP-UP study in that we did not use an automatic voice response system;rather, wecalled the patients individually, which might have led to a more aggressive lowering of the SUA in our trial. Furthermore, our observation period was longer, and a rheumatology provider performedthe intervention.

In a study by Doherty et al. (8), a nurse-led intervention was successful in obtaining a goal SUA $\leq 6 \mathrm{mg} / \mathrm{dl}$ in $95 \%$ of the patients after 2 years. Of note, in that study, telephone contact could be substituted for faceto-face visits, and home visits were permitted.In our study, patients were mostly newly diagnosed, which requires frequent adjustments in medication doses and frequent encounters, while in the study byDoherty et al., patients were mainly established patients with a mean duration of disease of 11 years. Furthermore, the higher percentage of patients achieving target SUA in the Doherty et al. study as compared to ours could be related to our rheumatology providers doing the intervention as part of their clinic duty to mimic real-life situations, while the study by Doherty et al. had an educated nursing staff dedicated to this study.Additionally, after entry into the telephone phase of theprogram, we did not conduct any face-to-face or home-visits as in the study by Doherty et al., which could also have led to higher percentage of patients achieving target SUA in their study versus ours.

A study by Yokose et al. (9), in which an e-visit conducted in 62 patients with gout was compared to a matched historic cohort, showed that SUA target of $\leq 6 \mathrm{mg} / \mathrm{dl}$ was obtained in $63 \%$ of patients in the intervention group versus $33 \%$ in the historic matched group. Our results were superior, likely because the Yokose et al. study used email reminders and questionnaires, while we conducted phone calls in real-time with patients.

There are several strengths toour study. Patients were enrolled after a visit with their rheumatology provider, which makesgout less likely to be misdiagnosed. We used real-time phone calls instead of automated contacts with patients which might have increased the rate of patient compliance. After usual 
face-to-face office visits, the patients were entered into a novel telephone phase of the program. This was very convenient to patients, which in turn led to higher patient satisfaction. We were able to achieve a goal SUA level $\leq 6 \mathrm{mg} / \mathrm{dl}$ in the majority of patients, and the rate of hospitalizations, ER, or urgent care visits due to gout was very low. We included a large number of patients in our study, and providers continued to refer patients to our study. This study was the first of its kind to employ telephone-based, guidelines-directed management prospectively led by rheumatology providers.

One limitation of this study was reaching patients by phone.There wereincidences where patients were not available by phone, and wewere forced to use our electronic medical record and mailing system to try to contact such patients. Additionally, repeated SUA levels were not always done on time after adjusting the medication dosage due to patient factors.Furthermore, we did not have a control group in our study, and as such we cannot draw definitive conclusions about the efficacy of this program as compared to standard of care. Furthermore, we performed the telephone encounters free of charge, but this may not be plausible at all institutions. There is also a possibility of enrollment bias as our patients were included based on the discretion of the initial rheumatology provider. Finally, there was no time-related data at entry on prior healthcare utilization.

Our study demonstrated that a gout disease management program consisting of typical visits with a rheumatologist followed by a novel telephone-based follow-up phase has a high patient satisfaction rate, and it may lead to improvement in following clinical guidelines, thus leading to better management of disease and reduced healthcare costs. Such visits can reduce outpatient office visits, with a more abbreviated management strategy by phone, thus freeing time to provide access to patients requiring bedside encounters. While providers were not reimbursed for doing such calls, new COVID-19 related changes in reimbursement for telemedicine care may change this in the future.Futureworkwith a comparable group that can assess the effect on healthcare expenditure is warranted to further elucidate the benefits of a GDMP for gout management.

\section{Declarations}

\section{Ethics approval and consent to participate}

This was determined to be an IRB-exempt project, therefore written patient consent was not obtained.

\section{Consent for publication}

NA

\section{Availability of data and materials}

The datasets during and/or analysed during the current study available from the corresponding author on reasonable request.

\section{Competing interests}


The authors declare that they have no competing interests

\section{Funding}

None

\section{Authors' contributions}

AA contributed to the acquisition, analysis, interpretation of data, drafted the work and substantively revised it. AA approved the submitted version.

TScontributed to the design, acquisition, analysis, interpretation of data and substantively revised the draft. TS approved the submitted version.

GL contributed to the acquisition and analysis of the data. GL approved the submitted version.

MCW contributed to the design, interpretation of data and made substantial revisions to the draft. MCW approved the submitted version.

WTA contributed to the design, acquisition, analysis, interpretation of data and substantively revised the draft. WTA approved the submitted version.

\section{References}

1. Singh JA, Hodges JS, Toscano JP, Asch SM. Quality of care for gout in the US needs improvement. Arthritis Rheum 2007;57: 822-9

2. Khanna D, Fitzgerald JD, Khanna PP, et al. 2012 American College of Rheumatology guidelines for management of gout. Part 1: systematic nonpharmacologic and pharmacologic therapeutic approaches to hyperuricemia. Arthritis Care Res 2012;64:1431-46. 10.1002/acr.21772

3. FitzGerald JD, Dalbeth N, Mikuls T, et al. 2020 American College of Rheumatology Guideline for the Management of Gout [published online ahead of print, 2020 May 11]. Arthritis Care Res (Hoboken). 2020;10.1002/acr.24180. doi:10.1002/acr.24180

4. Qaseem A, Harris RP, Forciea MA, et al. Management of acute and recurrent gout: a clinical practice guideline from the American College of Physicians. Ann Intern Med 2017;166:58-68. 10.7326/M160570

5. Jamal AB, Salma AH, Wafa AS, Ghadah A, Roaa A. The prescription of allopurinol in a tertiary care centre: appropriate indications and dose adjustment. Clin Med Insights Arthritis MusculoskeletDisord. 2012;5:53-57. doi:10.4137/CMAMD.S9803

6. Harrold LR, Andrade SE, Briesacher BA, Raebel MA, Fouayzi H, Yood RA, et al. Adherence with uratelowering therapies for the treatment of gout. Arthritis Res Ther 2009;11:R46.

7. Mikuls TR, Cheetham TC, Levy GD, Rashid N, Kerimian A, Low KJ, Coburn BW, Redden DT, Saag KG, Foster PJ, Chen L, R Curtis J. Adherence and Outcomes with Urate-Lowering Therapy: A Site- 
Randomized Trial. Am J Med. 2019 Mar;132(3):354- 361. doi: 10.1016/j.amjmed.2018.11.011. Epub 2018 Nov 29

8. Doherty M, Jenkins W, Richardson H, et al. Efficacy and cost-effectiveness of nurse-led care involving education and engagement of patients and a treat-to-target urate-lowering strategy versus usual care for gout: a randomised controlled trial. Lancet. 2018;392:1403-1412.

9. ChioYokose MD , April Jorge MD , Kristin D'Silva MD , Naomi Serling-Boyd MD , Mark Matza MD, MBA, Mazen Nasrallah MD, MS , Sarah Keller MD , Amar Oza MD , Hyon Choi MD, DrPH, Marcy B. Bolster MD , Deborah Collier MD , Using Electronic Visits (E-visits) to Achieve Goal Serum Urate Levels in Patients with Gout in a Rheumatology Practice: A Pilot Study, Seminars in Arthritis \& Rheumatism (2020), doi: https://doi.org/10.1016/j.semarthrit.2020.03.001

\section{Figures}

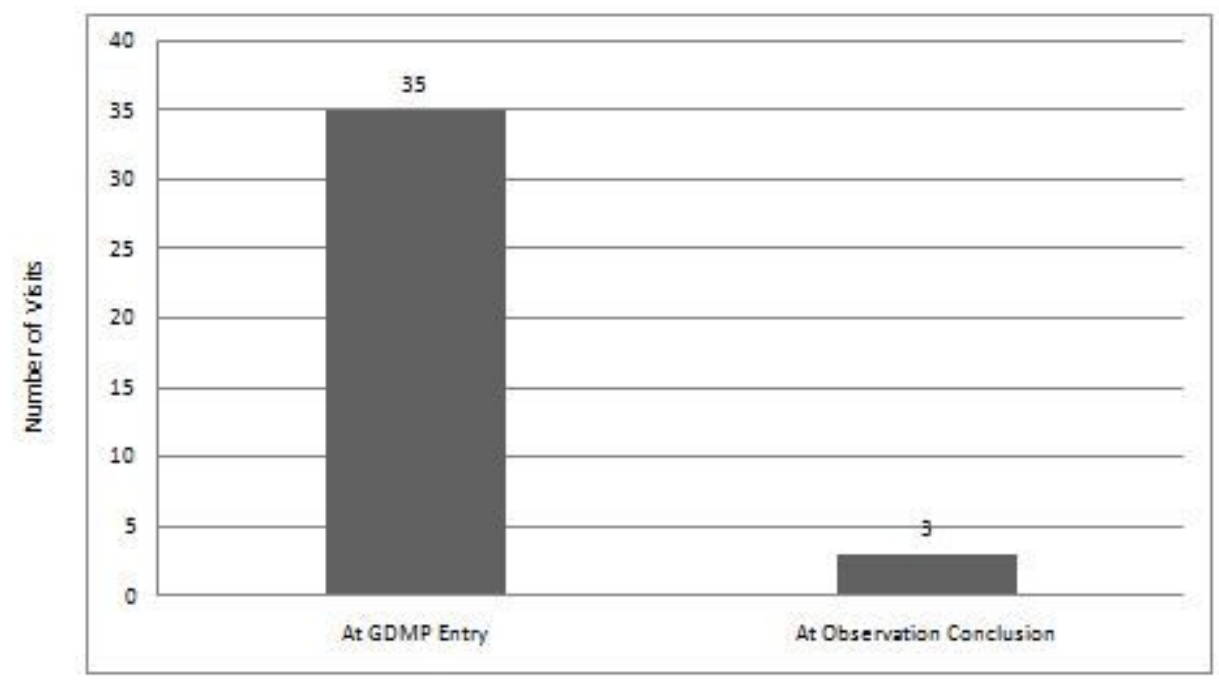

\section{Figure 1}

Hospitalization, ER, or Urgent Care Visits Due to Gout 


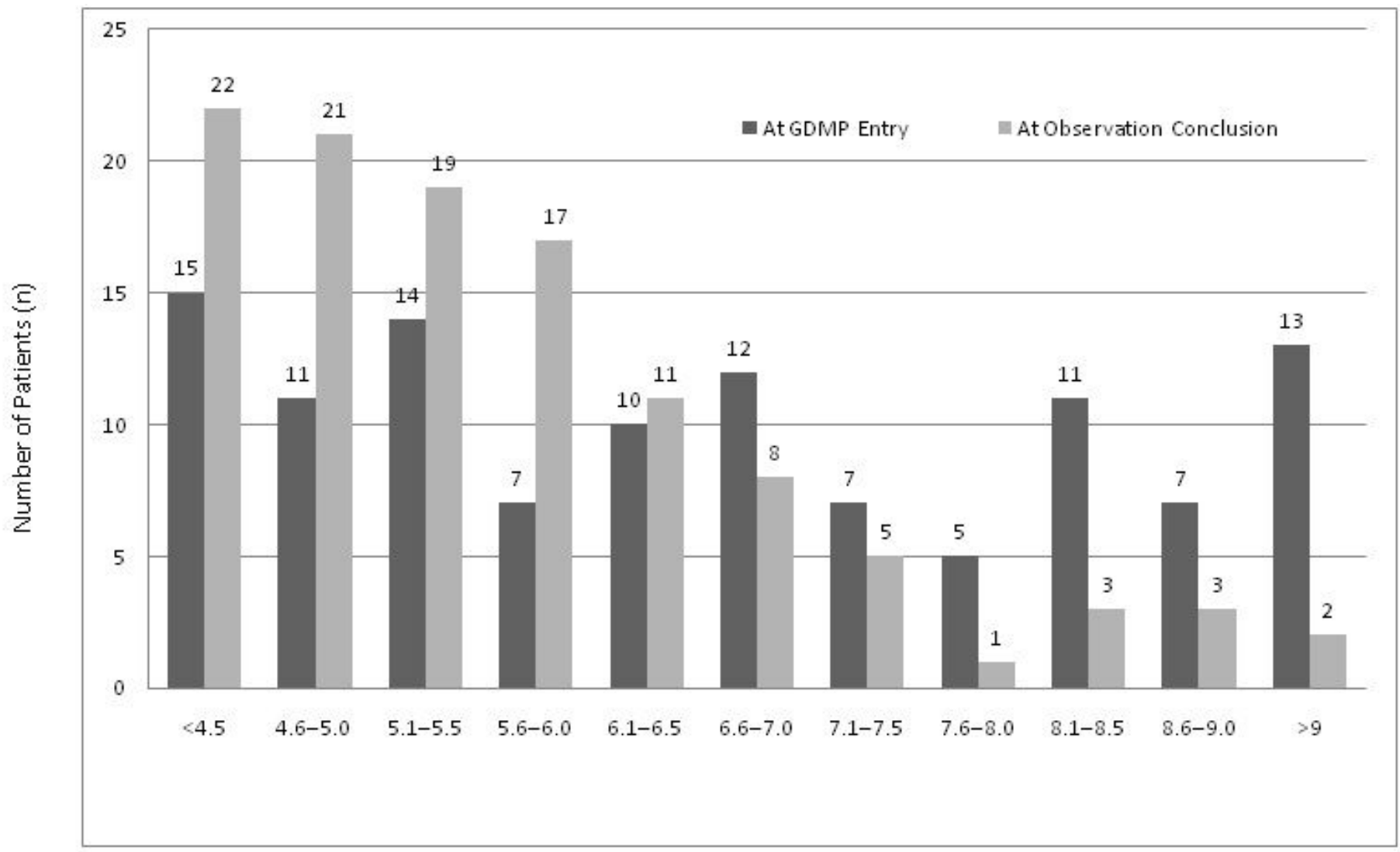

Figure 2

Distribution of SUA Levels at GDMP Entry and Observation Conclusion

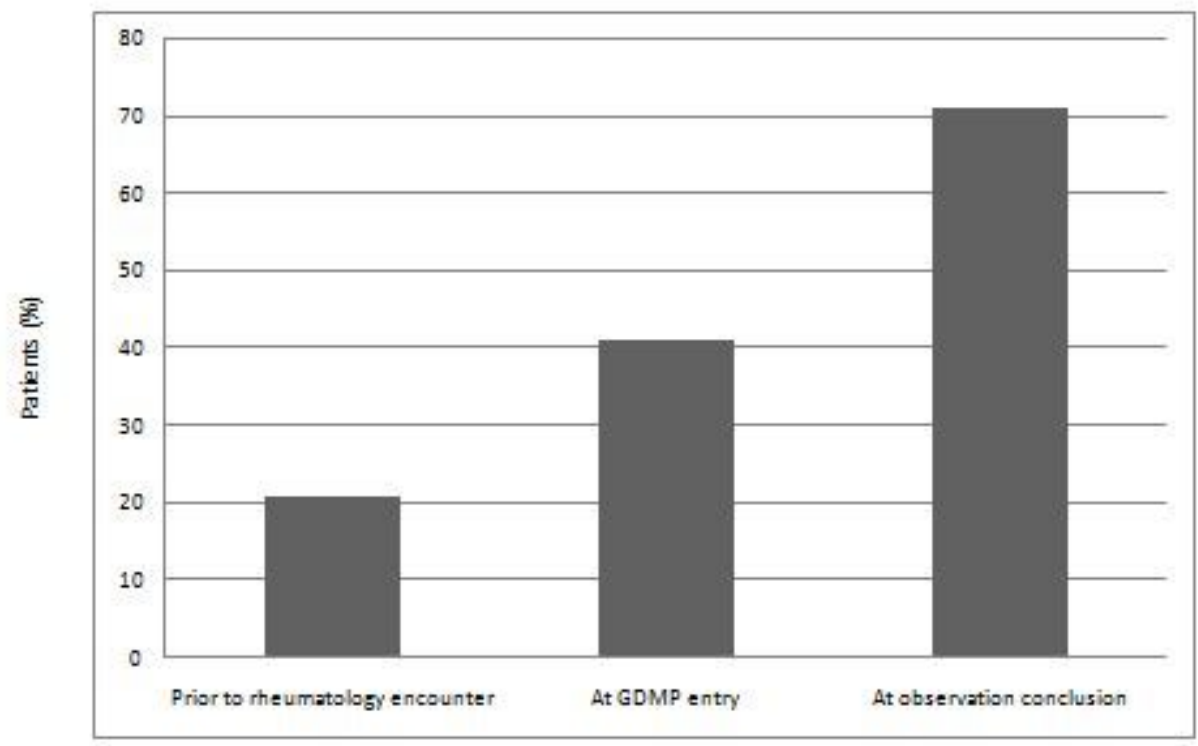

Figure 3

Percentage of Patients Achieving Goal SUA of $\leq 6 \mathrm{mg} / \mathrm{dl}$ 